\title{
Fiber-coupled NbN superconducting single-photon detectors for quantum correlation measurements
}

\author{
W. Slysz, ${ }^{* a}$ M. Wegrzecki, ${ }^{a}$ J. Bar, ${ }^{a}$ P. Grabiec, ${ }^{a}$ M. Gorska, ${ }^{a}$ E. Reiger, ${ }^{b}$ S. Dorenbos, ${ }^{b}$ V. Zwiller, ${ }^{b}$ I. Milostnaya, ${ }^{c}$ \\ O. Minaeva ${ }^{c}$ A. Antipov, ${ }^{c}$ O. Okunev, ${ }^{\mathrm{c}}$ A. Korneev, ${ }^{\mathrm{c}}$ K. Smirnov, ${ }^{\mathrm{c}}$ B. Voronov. ${ }^{\mathrm{c}}$ N. Kaurova, ${ }^{\mathrm{c}}$ G. N. Gol'tsman, ${ }^{\mathrm{c}}$ \\ J. Kitaygorsky, ${ }^{\mathrm{d}}$ D. Pan, ${ }^{\mathrm{d}}$ A. Pearlman, ${ }^{\mathrm{d}}$ A. Cross, ${ }^{\mathrm{d}}$ I. Komissarov, ${ }^{\mathrm{d}}$ and Roman Sobolewski ${ }^{\mathrm{d}}$ \\ ${ }^{a}$ Institute of Electron Technology, PL-02-668 Warszawa, Poland \\ ${ }^{\mathrm{b}}$ Delft University of Technology, 2600 GA Delft, The Netherlands \\ ${ }^{\mathrm{c}}$ Moscow State Pedagogical University, Moscow 119435, Russia \\ ${ }^{\mathrm{d}}$ University of Rochester, Rochester, NY 14627-0231, USA
}

\begin{abstract}
We have fabricated fiber-coupled superconducting single-photon detectors (SSPDs), designed for quantum-correlationtype experiments. The SSPDs are nanostructured ( $100-\mathrm{nm}$ wide and 4-nm thick) $\mathrm{NbN}$ superconducting meandering stripes, operated in the 2 to $4.2 \mathrm{~K}$ temperature range, and known for ultrafast and efficient detection of visible to nearinfrared photons with almost negligible dark counts. Our latest devices are pigtailed structures with coupling between the SSPD structure and a single-mode optical fiber achieved using a micromechanical photoresist ring placed directly over the meander. The above arrangement withstands repetitive thermal cycling between liquid helium and room temperature, and we can reach the coupling efficiency of up to $\sim 33 \%$. The system quantum efficiency, measured as the ratio of the photons counted by SSPD to the total number of photons coupled into the fiber, in our early devices was found to be around $0.3 \%$ and $1 \%$ for $1.55 \mu \mathrm{m}$ and $0.9 \mu \mathrm{m}$ photon wavelengths, respectively. The photon counting rate exceeded $250 \mathrm{MHz}$. The receiver with two SSPDs, each individually biased, was placed inside a transport, 60-liter liquid helium Dewar, assuring uninterrupted operation for over 2 months. Since the receiver's optical and electrical connections are at room temperature, the set-up is suitable for any applications, where single-photon counting capability and fast count rates are desired. In our case, it was implemented for photon correlation experiments. The receiver response time, measured as a second-order photon cross-correlation function, was found to be below $400 \mathrm{ps,} \mathrm{with}$ timing jitter of less than $40 \mathrm{ps}$.
\end{abstract}

Keywords: superconducting single photon detectors, single photon detectors, fiber-coupled optical detectors, quantum correlations, superconducting devices

\section{INTRODUCTION}

Modern quantum engineering is currently an area of science that is under intense investigation. Practical quantum systems such as quantum communications (QC) or quantum measurement systems require detectors with high speed, high sensitivity, high quantum efficiency (QE), and short dead times, along with precise timing characteristics and low dark counts. Superconducting single-photon detectors (SSPDs) are a new and highly promising type of devices fulfilling the above requirements [1]. A vast number of scientific work shows that SSPDs outperform traditional SPDs such as avalanche photodiodes (APDs) and photomultiplier tubes [2,3].

The most popular APDs have a limited utility for quantum applications [4]. Silicon APDs have their sensitivity restricted by the Si bandgap $(1.1 \mathrm{eV})$, therefore, they cannot be directly implemented in fiber-optic communication networks. InGaAs APDs work in the near infrared (NIR) region, and the best devices (in the 1.2 to $1.6 \mu \mathrm{m}$ wavelength range) have a QE $\sim 25 \%$ in single-photon detection mode, but suffer from very high $(>10 \mathrm{kHz})$ dark counts [5,6]. All APDs have to be gated and either passively or actively quenched to reduce the dark-count noise.

Presently, the most promising detectors are two types of SSPDs, one of which is the transition-edge sensor (TES) [7]. The TES is a superconducting tungsten bolometer and, because of its extreme high-energy sensitivity, it exhibits photon number resolving capabilities and very high QE (> 86\%). It operates at temperatures $T<100 \mathrm{mK}$ and has very low 
$(<0.03 \mathrm{~Hz})$ dark-count rates [7]. These devices, however, suffer greatly from a $4-\mu$ s recovery time and a 72-ns full width at half-maximum (FWHM) arrival time resolution (jitter).

Another type of the SSPD, recently developed and presented here, is an ultrathin, submicron-width NbN nanostructure maintained at $T$ far below the $\mathrm{NbN}$ critical temperature $T_{c}[1,2]$. The SSPD operation principle has been explained within a phenomenological hot-electron photoresponse model $[9,10]$. As we have already stated, the NbN SSPDs are ultrafast, and efficiently count photons with wavelengths ranging from ultraviolet to infrared. They have already been successfully implemented for free-space (laboratory) detection at wavelengths 0.4 to $6.0 \mu \mathrm{m}[2,16]$, as well as in optical fiber-based setup at wavelengths $0.9,1.3$, and $1.55 \mu \mathrm{m}[11,12]$.

Fiber-coupled SSPDs are very easy to implement into optics experiments. The first practical application of these detectors was for non-invasive testing and debugging of CMOS integrated circuits [13,14], while recently, they were implemented for fast lifetime measurements of quantum well structures emitting IR radiation [15], and for determination of spontaneous emission lifetimes of InAs quantum dot single photon sources [3]. Our SSPDs are also very attractive for QC protocols due to low dark counts, in combination with the fast recovery time, and hence, high maximal counting rate [16].

In this work, we present the results of an experiment with two fiber-coupled SSPDs in a Hanbury-Brown and Twiss interferometer measurement of the source second-order correlation function $g^{(2)}$. We used a $0.9-\mu \mathrm{m}$-wavelength femtosecond pulsed laser as a test source. The fiber-coupled $\mathrm{NbN}$ detectors work immersed in liquid helium, and since both their fiber and electrical interconnects are outside the Dewar, they can be regarded as a room-temperature-like devices $[11,12]$.

\section{CONSTRUCTION OF THE DETECTOR}

The NbN SSPDs were fabricated according to a technological procedure described in detail in [17]. Most of the detectors used in our receivers were $10 \times 10 \mu \mathrm{m}^{2}$, NbN meander-type structures with a 4-nm film thickness and a 120 $\mathrm{nm}$ nominal strip width, as shown in Fig. 1. Recently, we have also used $10 \times 20 \mu \mathrm{m}^{2}$ devices. The critical current density $J_{C}$ of the completed nanostructures varied from 2 to $6 \mathrm{MA} / \mathrm{cm}^{2}$ at $4.2 \mathrm{~K}$, with $T_{C} \approx 10 \mathrm{~K}$. The basic idea of the fiber-coupled SSPD working immersed and operated in a standard liquid-helium transport Dewar has been presented in our previous works $[11,12]$.

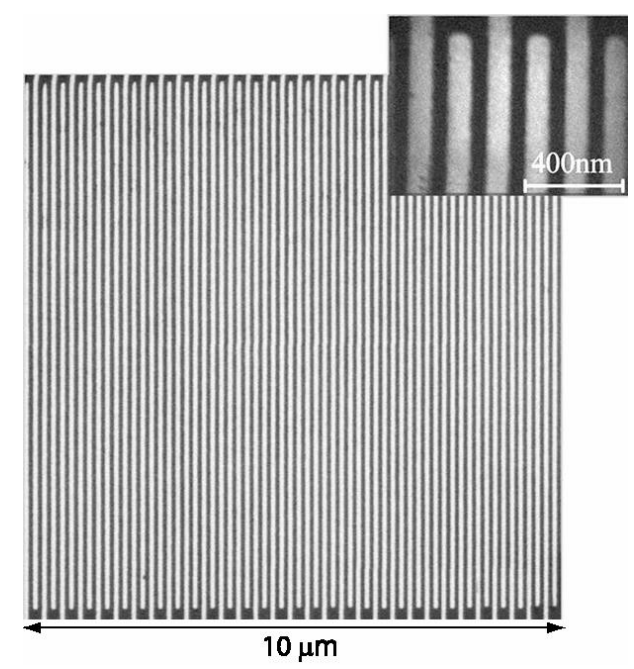

Fig. 1. SEM image showing the active area (meander) of the NbN SSPD device (NbN is black). 
The photon delivery to the $\mathrm{NbN}$ detector is provided by a single or multimode fiber, while the electrical output is connected to room-temperature electronics via an SMA connector directly integrated with the SSPDs coplanar waveguide output. A picture of the copper flange with the $\mathrm{NbN}$ structure and both the fiber and electrical connections is shown in Fig. 2. Figure 2(a) demonstrates a single-SSPD receiver, while in Fig. 2(b) we illustrate an integrated twodetector arrangement.

For coupling between the $\mathrm{NbN}$ structure and the fiber, we used a specially designed micromechanical photoresist ring positioned directly over the SSPD. The coupling rings were fabricated using a photolithography process guided by alignment marks made on the structure in the fabrication process [18]. The positioning accuracy of the ring was $1 \mu \mathrm{m}$. Figure 3 shows the fiber, stripped of its jacket and pushed against the $\mathrm{NbN}$ film, and permanently attached with optical glue. Figure 4 illustrates the geometry of the fiber-detector arrangement. The calculations of coupling efficiency, presented in [19], show that we can expect the coupling values from 20 to $90 \%$ for single-mode fibers.

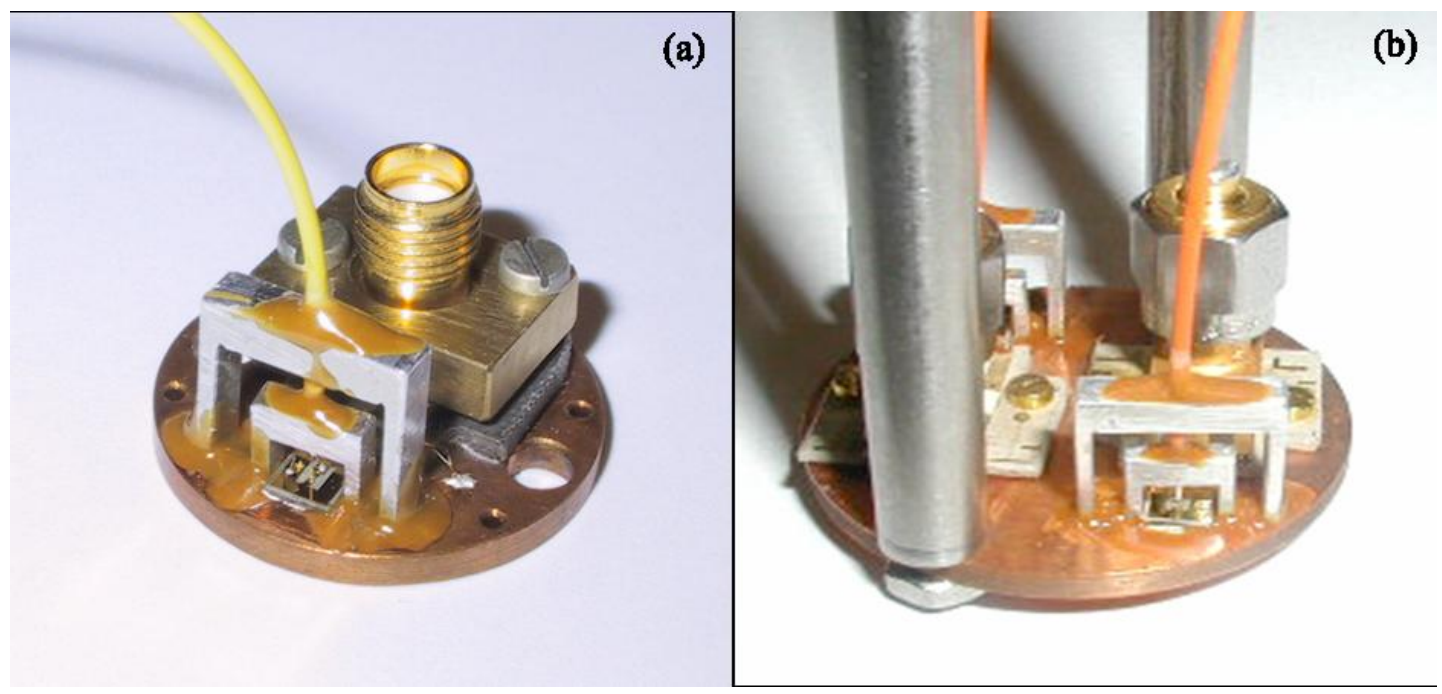

Fig. 2. Copper flange with (a) single-SSPD setup and (b) two-detector setup (two-channel device).

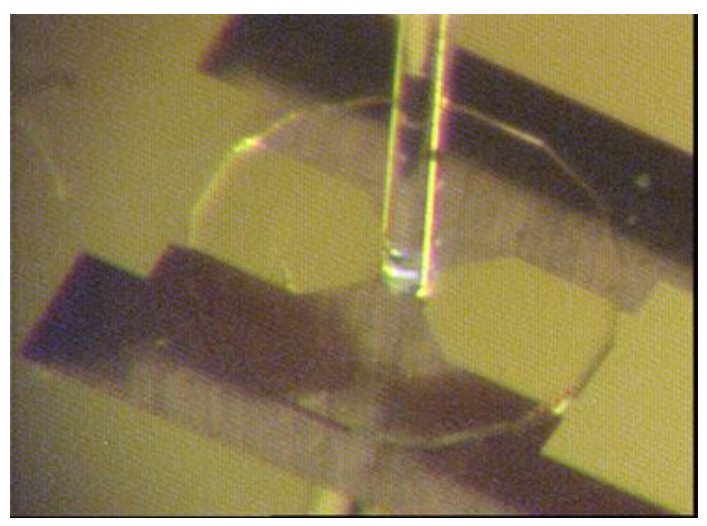

Fig. 3. The photoresist coupling ring and the fiber glued on the top of the detector structure. 


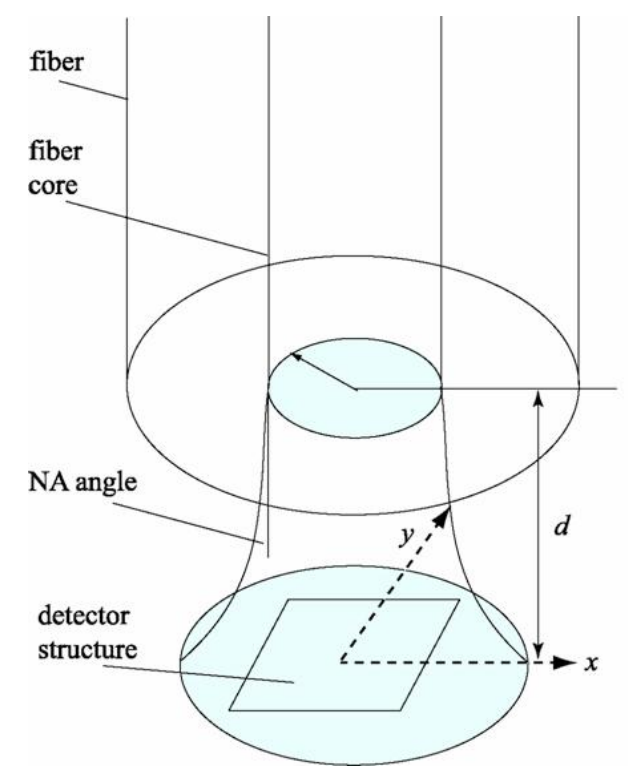

Fig. 4. Schematic geometry of the fiber-coupled detector system.

\section{EXPERIMENTAL RESULTS}

\subsection{System quantum efficiency}

We have fabricated several $\mathrm{NbN}$ detectors coupled via single-mode fibers, and several with multimode fibers. The main figure of merit was the system QE (SQE) for each device [2,19,20]. We used highly attenuated, 41-ps-wide pulses from a semiconductor laser diode operating at a $1.54 \mu \mathrm{m}$ wavelength with a repetition rate of $1 \mathrm{MHz}$. The optical power delivered to the detectors was controlled with a digital optical attenuator. We measured the optical power at the end of the fiber before its entering into the cryostat. The power level for each measuring point was calculated using the attenuation values. The optical power values were divided through the value of the photon energy, in order to determine the photon flux level in photons/second. The number of photons per second was divided through the repetition rate value $(1 \mathrm{MHz})$, to get the average number of photons in one pulse. The output signals from the amplifiers inside the electronics boxes were connected to either a fast oscilloscope for time-resolved studies, or to a signal counter to perform statistical analysis of our photon counts or to measure dark counts.

We define QE as the ratio of the SSPD photoresponse counts, $N_{\text {resp }}$ to the number photons provided to the detector, $N_{\text {inc }}$ :

$$
Q E=N_{\text {resp }} / N_{\text {inc }}
$$

When we define $N_{i n c}$ as a number of photons measured at room temperature in the fiber in our setup, we directly deal SQE. Sometimes, we take the number of photons falling on the SSPD active area as $N_{\text {inc }}$. This latter value is derived from the power measurements in the fiber setup and from the geometry of the detector, taking into account the detector active area and the area illuminated by the fiber (see Fig. 4). In case case, we are talking about QE of the detector structure:

$$
Q E_{\text {structure }}=N_{\text {resp }} / N_{\text {fall }} \text {. }
$$


For single-mode fiber-coupled detectors, we typically discuss SQE, as the most important parameter for the user. For multimode fibers, because of their large core area $\left(2000 \mu \mathrm{m}^{2}\right)$, we can also talk about the $\mathrm{QE}_{\text {structure }}$, since only a small portion of radiation reaches the detector with a much smaller $\left(100 \mu \mathrm{m}^{2}\right)$ active area.

Typical dependence of SQE versus normalized bias current for the incident power, expressed as 1 photon per optical pulse incident on the $\mathrm{NbN}$ detector, is presented in Fig. 5. The SQE was measured to be $0.33 \%$ at $1.55 \mu \mathrm{m}$ wavelength, and the coupling efficiency was calculated to be $\sim 30 \%$.

The inset in Fig. 5 shows the dark counting rate $R_{d k}$ for our SSPDs. The dark counts are manifested as spontaneous voltage pulses similar to the photoresponse pulses while the detector is blocked from all incoming radiation. We can see that for higher currents, detector has both higher efficiency and higher $R_{d k}$, and both dependencies were explained in more detail in our previous work [12,21]. We describe the trade off between $\mathrm{QE}$ and $R_{d k}$ in terms of the noise equivalent power NEP, which is defined for quantum detectors as:

$$
N E P=\frac{h v}{S Q E} \sqrt{2 R_{d k}}
$$

where $h v$ is the photon energy. To determine the NEP (also shown in Fig. 5), we used the measured values of SQE and $R_{d k}$. We can see that the lowest value of NEP is for $I_{b} / I_{c}$ between 0.9 and 0.95 . The obtained results of NEP for our detectors measured at $4.2 \mathrm{~K}$ are already good, and we can expect that for $T=2 \mathrm{~K}$ the results will be even better [21]. Similar $\mathrm{NbN}$ detectors produced from the same wafers have QE values up to 5 times higher, and $R_{d k}$ values up to 100 times lower, so for $T=2 \mathrm{~K}$ we can expect the NEP values to be $\sim 3$ orders of magnitude lower.

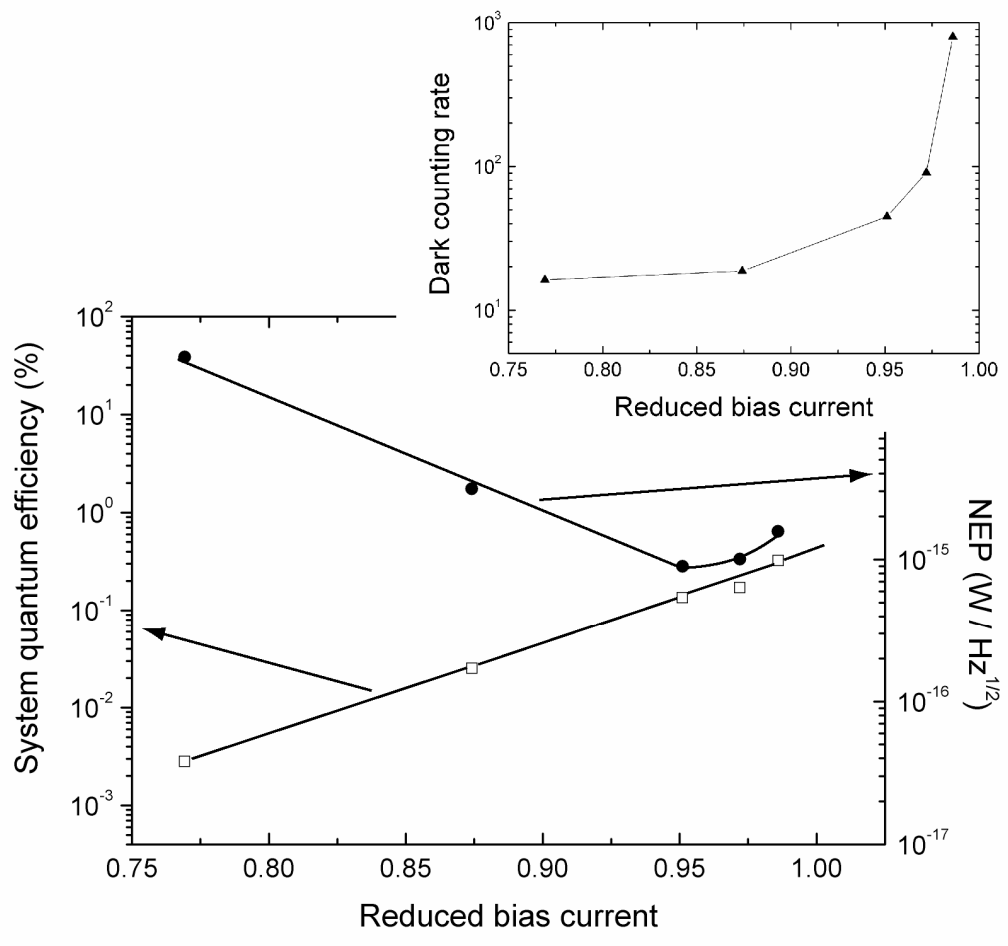

Fig. 5. System quantum efficiency (open sybbols, left axis), noise equivalent power (NEP) (closed symbols, right axis), and the dark counting rate (inset) vs. normalized biased current for one of the best manufactured fiber-coupled detectors, measured at $T=4.2 \mathrm{~K}$ and $1.55 \mu \mathrm{m}$ wavelength. Solid lined are guides to the eye. 
Figure 6 compares the spectral dependence of SQE for two fiber-coupled SSPDs with our earlier QE measurement of a free-space-coupled SSPD [2]. All three data sets follow exponential dependencies, meaning that, in all devices, the photoresponse mechanism is the same, and is based on the hotspot formation and current redistribution model $[9,10]$. We also note that the two data sets corresponding to our fiber-coupled devices exhibit essentially the same slope, and differences are observed in the actual QE values. In the case of our earlier, 3.5-nm-thick, free-space device, the QE versus the wavelength dependence tends to reach saturation at short wavelengths, but we must remember that this is actually the $\mathrm{QE}_{\text {structure }}$ value, where the saturation corresponds to the light-absorption coefficient $(\sim 20 \%)$ of a 3.5-nmthick $\mathrm{NbN}$ film. The lack of saturation for fiber-coupled devices, even at 400-nm wavelengths, is, apparently, due to the fact that $\mathrm{SQE}<<\mathrm{QE}_{\text {structure }}$ for our tested detectors.

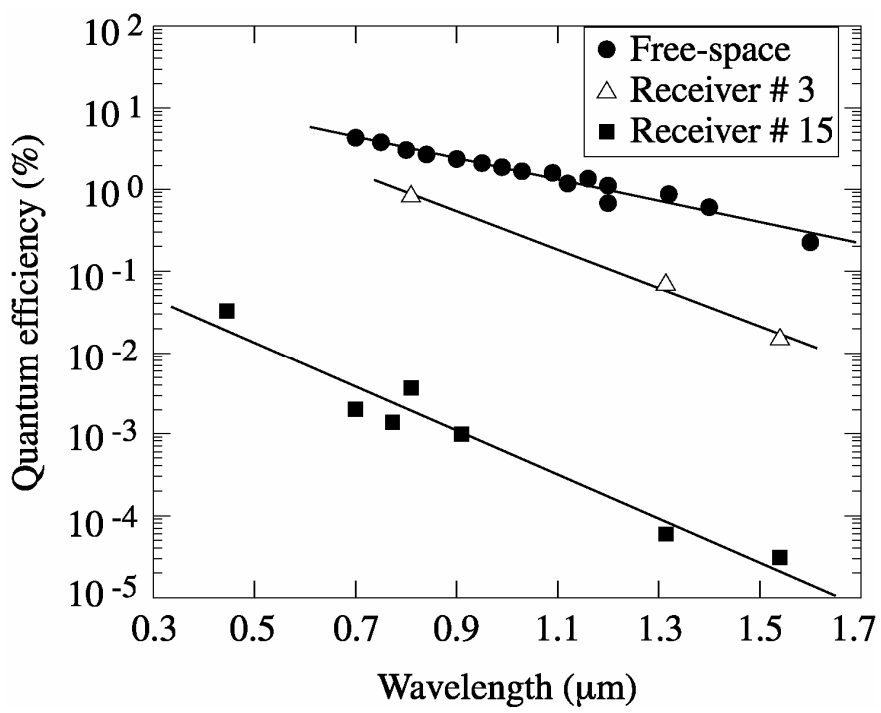

Fig. 6. The spectral dependence of SQE for two fiber-coupled detectors (receiver \#15 and receiver \#3), compared with our earlier QE measurements of a free-space-coupled SSPD.

\subsection{Photoresponse parameters}

As it was demonstrated by Kerman et al. [22], for large-area, meander-type SSPDs, the photoresponse pulse width and the corresponding fall time are limited by a large kinetic inductance of the meander structure. Setups that consisted of a bias-tee $(80 \mathrm{kHz}$ to $26 \mathrm{GHz})$ semiconductor laser $(\lambda=1.55 \mu \mathrm{m})$, a broadband amplifier $(50 \mathrm{MHz}$ to $4 \mathrm{GHz})$, and a $6-$ $\mathrm{GHz}$, Tektronix, single-shot digital oscilloscope were used to measure the photoresponse time of our fiber-coupled detectors. We measured a 250-ps rise time and a 5-ns fall time with a FWHM of about 2.5 ns, as shown in Fig. 7 (dotted line). This figure also shows a typical dark-count pulse (gray line), observed with the fiber input completely blocked. We note that the shape of the dark-count pulse is identical with the photoresponse pulse, confirming that the SSPD output signal was limited by the large, intrinsic kinetic inductance.

The above observation was directly confirmed by our theoretical calculations (black solid line in Fig. 7) of a photoresponse of a $100-\mu \mathrm{m}^{2}$-area SSPD, taking into account the true dimensions of the NbN SSPD: thickness $d=4 \mathrm{~nm}$, stripe width $w=120 \mathrm{~nm}$, and an 80-nm gap between the stripes [11]. 


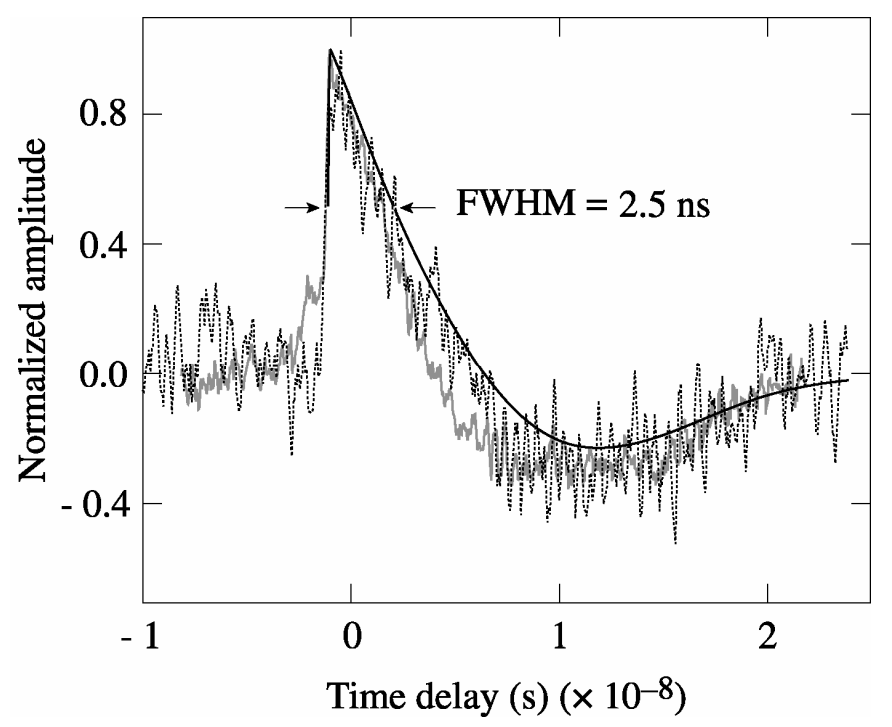

Fig. 7. Measured photoresponse signal (dotted line) and dark response (gray solid line) of a $10 \times 10 \mu \mathrm{m}^{2} \mathrm{NbN}$ detector and compared with theoretical calculation (black solid line).

In the same experimental setup, we also measured the timing jitter of our fiber-coupled detectors. The experimental data are shown in Fig. 8, and were collected using a standard histogram feature installed in our 50-GHz Tektronix sampling oscilloscope. For comparison, we also included the timing fitting data of one of our older, free-space-coupled SSPDs [2]. We note that the jitter profile in all cases is Gaussian and for fiber-coupled structures, the FWHM varies from $37 \mathrm{ps}$ for a single-mode-fiber detector to $60 \mathrm{ps}$ for a multimode one. In both cases, the jitter is higher than the for the early SSPD measured in a free-space arrangement, apparently, because of the undesired impact of $\sim 2$-m-long fibers and electrical output cables implemented in our presented here cryogenic setup. Finally, for the detector \#3, coupled via a multimode fiber, we have to include modal dispersion in our estimation, which itself can account for $\geq 24$ ps of dispersion in standard fibers of $\sim 2 \mathrm{~m}$ [23].

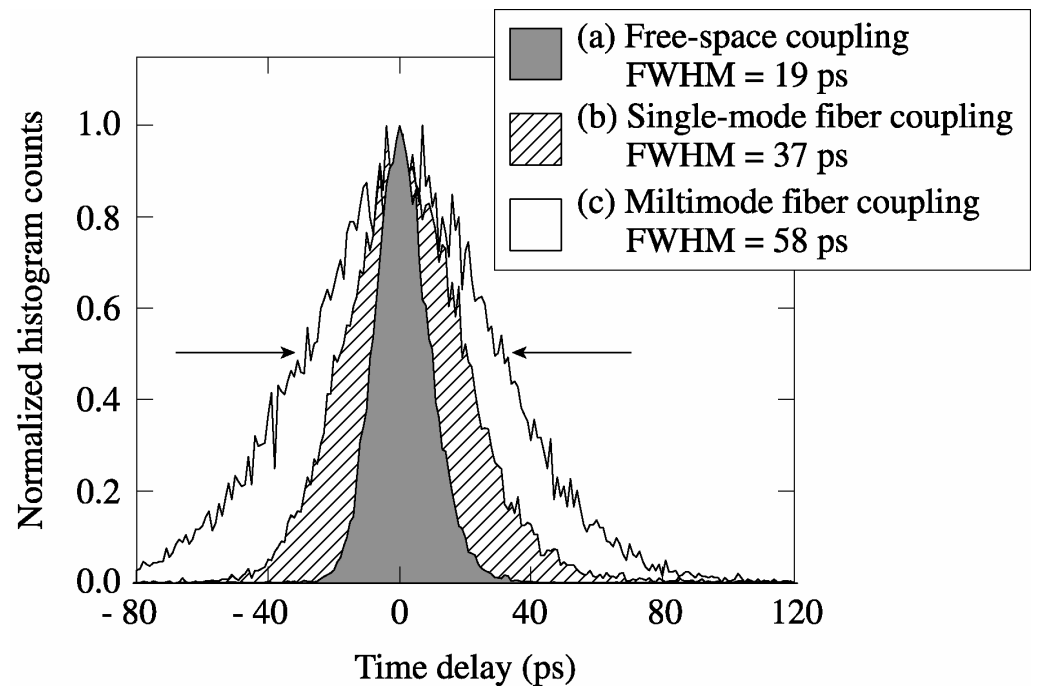

Fig. 8. Jitter comparison of NbN SSPDs at a 1540-nm wavelength. (a) $4-\times 4-\mu \mathrm{m}^{2}$ small $L_{\text {kin }}$ device with free-space coupling and a jitter of 19-ps FWHM. (b) A 10- $\times 10-\mu \mathrm{m}^{2}$ large $L_{\text {kin }}$ device with a single-mode fiber and a jitter of 37-ps FWHM. (c) A $10-\times 20-\mathrm{m}^{2}$ large $L_{\text {kin }}$ device with a multimode fiber and a jitter of 58-ps FWHM. 


\subsection{Correlation measurements}

To further test the timing resolution of our complete receiver systems and determine their usefulness for applications in photon quantum-correlation experiments, we carried out our own correlation experiments with two SSPD detectors integrated on a single flange [see Fig. 2(b)]. As is shown in Fig. 9, light from a fiber-coupled, pulsed-laser source was attenuated and split by a 50/50 beam splitter, and, subsequently, detected by our two SSPDs. The signal from each detector was fed to a "start-stop" time correlator and could be plotted as function of a delay between arrival times of photons at each detector. As a result, we could directly obtain a second-order photon cross-correlation function $g^{(2)}$, which is presented in Fig. 10. For our tests, we used 500-fs-wide pulses at $\lambda=940 \mathrm{~nm}$ with a repetition rate of $82 \mathrm{MHz}$. In Fig. 10(a), the detector \#12 with a low SQE ( 0.005\%) operated as a start device and the high SQE detector \#11 acted as a stop. The presented correlation time, FWHM of $390 \mathrm{ps,} \mathrm{can} \mathrm{be} \mathrm{regarded} \mathrm{as} \mathrm{the} \mathrm{time} \mathrm{resolution} \mathrm{of} \mathrm{the}$ complete correlation system (amplifiers, discriminator, correlator, cables, terminators, and detectors).

Figure 10(b) shows the correlation measurements with the start and stop detectors reversed. As expected, the correlation widths are virtually identical (430-ps FWHM) and only the total number of counts decreases. The widths of the correlation traces shown are relatively narrow compared to standard APD quantum correlation systems, but wider compared to a combination APD-SSPD system presented in [3]. The latter results demonstrate the utility of SSPDs in general, and our fiber-coupled devices in particular for quantum-correlation measurements [24]. Despite a few nanosecond-long response times of our detectors, the correlation pulses are subnanosecond. The latter, we believe, is due to the very low jitter and the short $(250 \mathrm{ps})$ photoresponse rise time of the SSPDs, and demonstrates the ability of our fiber-coupled detectors to successfully perform antibunching-type correlation experiments.

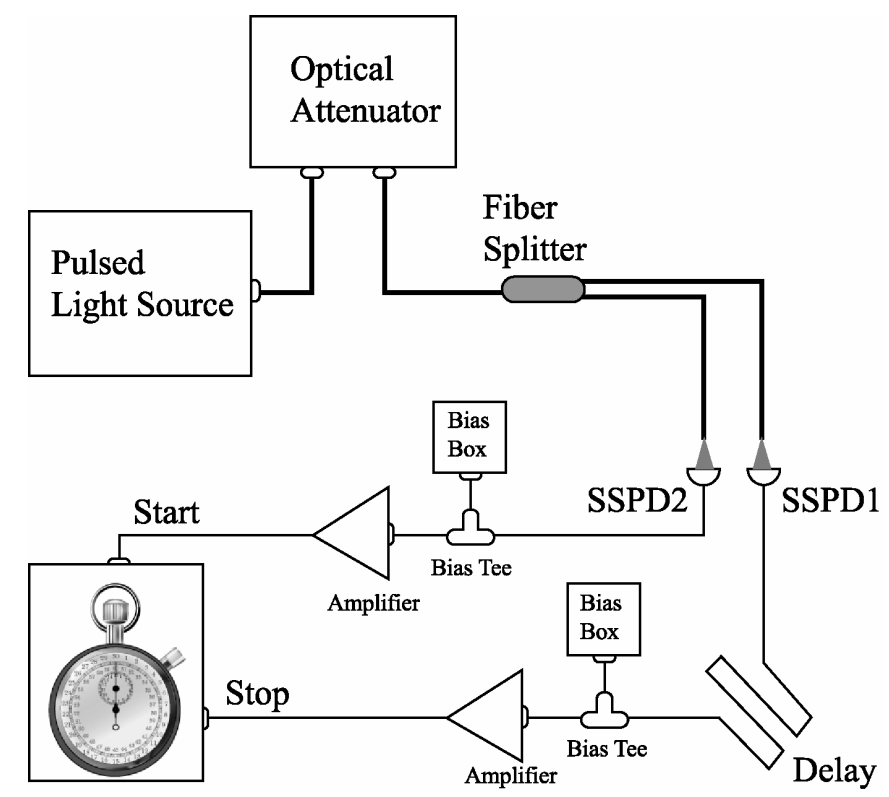

Fig. 9. Experimental setup for our photon-correlation measurements. Light source was a MIRA 900 laser, while photon correlations were measured using a TimeHarp time card. 
(a) Start: device \#12, stop: device \#11

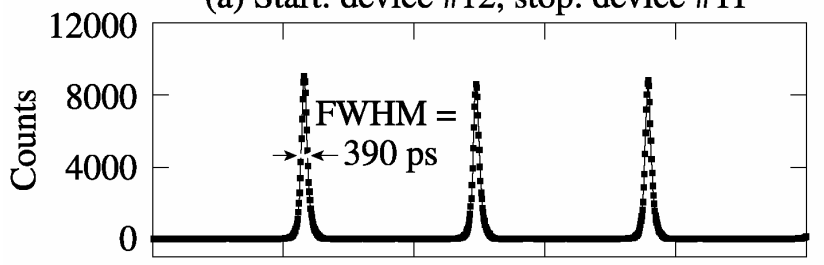

(b) Start: device \#11, stop: device \#12

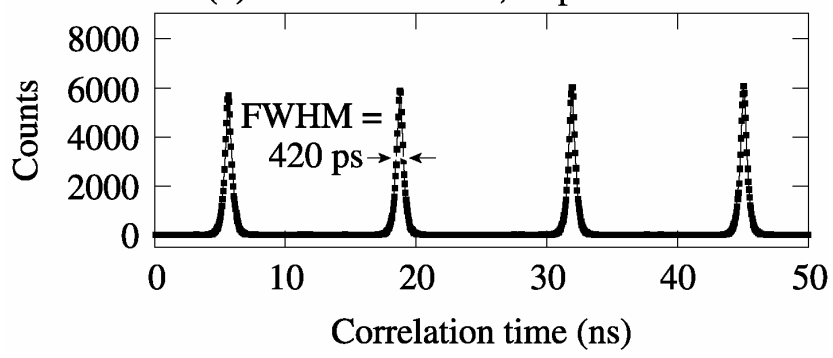

Fig. 10. Correlation measurements on 82-MHz laser pulses with NbN SSPDs. (a) High-SQE device \#11 as a stop detector. (b) Low-SQE device \#12 as a stop detector.

\section{Conclusions}

We have fabricated and tested a number of fiber-based, single-photon detectors designed for applications in practical quantum engineering systems. The SQE of our best detector was measured as $0.33 \%$ and coupling efficiency at $30 \%$, however, we expect to reach values as high as 3\% to 5\%. This will be accomplished by implementing SSPDs with higher $\mathrm{QE}_{\text {structure }}$ values, since the estimated coupling efficiency of the fiber-detector setup in our design has already reached 30\%. Microlenses and thicker (e.g., 50- $\mu \mathrm{m}$-thick) photoresist rings should increase the coupling efficiency even further.

Our devices were placed inside the sealed helium dewar with SSPDs maintained at $T=4.2 \mathrm{~K}$, while the optical and electrical connections remained at room temperature. Therefore, the setup can be regarded as a "room-temperature-like" system by an external user. The detector performance parameters presented above make our receivers especially useful for such applications as practical quantum key distribution and photon quantum-correlation measurements.

We believe that we demonstrated the ability of our fiber-coupled detectors to successfully perform antibunching-type correlations observed in photon emitted by true single-photon sources, such as single quantum dots.

\section{Acknowledgement}

The authors would like to thank Pascal Bohi for his participation in time-correlation measurements. The work in Moscow was supported by the grant "Non-equilibrium processes after IR photon absorption in thin-film superconducting nanostructures" of Russian Agency on education and the grant 02.445.11.7434 of Russian Ministry of education and science for support of leading scientific schools, INTAS project 03-51-4145, and by the European Commission under project "SINPHONIA", contract number NMP4-CT-2005-16433. Research conducted in Rochester was supported in part by the U.S. AFOSR Grant FA9550-06-1-0348 and the NYSTAR grant to the University of Rochester CAT-EIS. Finally, the Delft group acknowledges support from the NWO foundation. 


\section{REFERENCES}

1. A Verevkin, A. Pearlman, W. Slysz, J. Zhang, M. Currie, A. Korneev, G. Chulkova, O. Okunev, P. Kouminov, K. Smirnov, B. Voronov, G. Gol'tsman, and R. Sobolewski, "Ultrafast superconducting single-photon detectors for near-infrared-wavelength quantum communication", J. Mod. Opt. 51, $1447-1458$ (2004).

2. A. Korneev, P. Kouminov, V. Matvienko, G. Chulkova, K. Smirnov, B. Voronov, G. N. Gol'tsman, M. Currie, W. Lo, K. Wilsher, J. Zhang, W. Słysz, A. Pearlman, A. Verevkin, and R. Sobolewski, "Sensitivity and gigahertz counting performance of NbN superconducting single-photon detectors," Appl. Phys. Lett.,(84), 5338 (2004).

3. R. H. Hadfield, M. J. Stevens, S. S. Gruber, A. J. Miller, R. E. Schwall, R. P. Mirin, and S. W. Nam, "Single photon source characterization with a superconducting single photon detector," Opt. Express, 13, 10846 (2005).

4. Kurtsiefer, C., Zarda, P., Halder, M., Gorman, P. M., Tapster, P. R., Rarity, J. G., and Weinfurter, H., 2002, in Quantum Optics in Computing and Communications, Liu, S., Guo, G., Lo, H.-K., and Imoto, N., Eds. (Bellingham, WA: SPIE), Vol. 4917, p. 25.

5. Y. Kang, Y.-H. Lo, M. Bitter, S. Kristjansson, Z. Pan, and A. Pauchard, "InGaAs-on-Si single photon avalanche photodetectors," Appl. Phys. Lett. 85)\, 1668 (2004).

6. G. Ribordy, N. Gisin, O. Guinnard, D. Stucki, M. Wegmuller, and H. Zbinden, "Photon counting at telecom wavelengths with commercial InGaAs/InP avalanche photodiodes: current performance," J. Mod. Opt. 51, 1381 (2004).

7. B. Cabrera, R. M. Clarke, P. Colling, A. J. Miller, S. Nam, R.W. and Romani, "Detection of single infrared, optical, and ultraviolet photons using superconducting transition edge sensors," Appl. Phys. Lett. 73, 735 (1998).

8. D. Rosenberg, S. W. Nam, P. A. Hiskett, C. G. Peterson, R. J. Hughes, J. E. Nordholt, A. E. Lita, and A. J. Miller, "Quantum key distribution at telecom wavelengths with noise-free detectors," Appl. Phys. Lett. 88, 021108 (2006).

9. G. N. Gol'tsman, O. Okunev, G. Chulkova, A. Lipatov, A. Semenov, K. Smirnov, B. Voronov, A. Dzardanov, C. Williams, and R. Sobolewski, "Picosecond superconducting single-photon optical detector," Appl. Phys. Lett. 79, 705-707 (2001).

10. A. D. Semenov, G. N. Gol'tsman, and A. A. Korneev, "Quantum detection by current carrying superconducting film," Physica C, 351, 349-356 (2001).

11. W. Słysz, M. Wegrzecki, J. Bar, P. Grabiec, M. Górska, V. Zwiller, C. Latta, P. Böhi, A. Pearlman, A. Cross, D. Pan, I. Komissarov, A. Verevkin, I. Milostnaya, A. Korneev, O. Minayeva, G. Chulkova, K. Smirnov, B. Voronov, G. Gol'tsman, M. Currie, and Roman Sobolewski, "Fibre-coupled, single-photon detector based on NbN superconducting nanostrucures for quantum communications," Special Issues on Single Photon: Sources, Detectors, Applications, and Measurement Method, J. Mod. Optics, 54, 356-326 (2007).

12. W. Słysz, M. Węgrzecki, J. Bar, P. Grabiec, M. Górska, V. Zwiller, C. Latta, P. Böhi, I. Milostnaya, O. Minaeva, A. Antipov, O. Okunev, A. Korneev, K. Smirnov, B. Voronov, N. Kaurova, G. Gol'tsman, A. Pearlman, A. Cross, I. Komissarov, A. Verevkin, i Roman Sobolewski, Appl. Phys. Lett. 88, 261113 (2006).

13. J. Zhang, N. Boiadjieva, G. Chulkova, H. Deslandes, G. N. Gol'tsman, A. Korneev, P. Kouminov, M. Leibowitz, W. Lo, R. Malinsky, O. Okunev, A. Pearlman, W. Słysz, A. Verevkin, K. Wilsher, C. Tsao, and R. Sobolewski, "Non-invasive CMOS circuit testing with $\mathrm{NbN}$ superconducting single-photon detectors," Elect. Lett. 39 (14), 1086 (2003).

14. S. Somani, S. Kasapi, K. Wilsher, W. Lo, R. Sobolewski, and G. Gol'tsman, "New photon detector for device analysis: Superconducting single-photon detector based on a hot electron effect," J. Vac. Sci. Tech. B 19, 2766 (2001).

15. M. J. Stevens, R. H. Hadfield, R. E. Schwall, S. W. Nam, R. P. Mirin, J. A. Gupta, "Fast lifetime measurements of infrared emitters using a low-jitter superconducting single-photon detector," Appl. Phys. Lett. 89, 031109, (2006).

16. A. Korneev, O. Mineava, A. Divochiy, A. Antipov, N. Kaurova, V. Seleznev, B. Voronov, G. Gol'tsman, D. Pan, J. Kitaygorsky, W. Slysz, R. Sobolewski, "Ultrafast and high quantum efficiency large-area superconducting singlephoton detectors," these proceedings.

17. G. N. Gol'tsman, K. Smirnov, P. Kouminov, B. Voronov, N. Kaurova, V. Drakinsky, J. Zhang, A. Verevkin, and R. Sobolewski, "Fabrication of nanostructured superconducting single-photon detectors," IEEE Trans. Appl. Supercond. 13, 192 (2003).

18. P. Grabiec, W. Slysz, M. Wegrzecki, J. Bar, W. Milczarek, G. N. Gol’tsman, A. Verevkin, and R. Sobolewski, Patent pending No P-367391, Poland (2005). 
19. W. Słysz, M. Węgrzecki, J. Bar, P. Grabiec, M. Górska, C. Latta, . V. Zwiller , A. Pearlman, A. Cross, A. Korneev, P. Kouminov, K. Smirnov, B. Voronov, G. Gol’tsman, A. Verevkin, M. Currie, and Roman Sobolewski, Proc SPIE vol. 5957 (2005).

20. A. Verevkin, J. Zhang, R. Sobolewski, A. Lipatov, O. Okunev, G. Chulkova, A. Korneev, K. Smirnov, G. N. Gol'tsman, and A. Semenov, "Detection efficiency of large-active-area $\mathrm{NbN}$ single-photon superconducting detectors in the ultraviolet to near-infrared range," Appl. Phys. Lett. 80, 4687 (2002).

21. G. Gol'tsman, O. Minaeva, A. Korneev, M. Tarkhov, Rubtsova, A. Divochiy, I. Milostnaya, G. Chulkova, N. Kaurova, B. Voronov, D. Pan, J. Kitaygorsky, A. Cross, A. Pearlman, I. Komissarov, W. Słysz, M. Węgrzecki, J. Bar, P. Grabiec, and Roman Sobolewski ,to be published in IEEE Trans. Appl. Supercond. (2007).

22. A. J. Kerman, E. A. Dauler, W. E. Keicher, J. K. W. Yang, K. K. Berggren, G. Gol'tsman, and B. Voronov, "Kinetic-inductance-limited reset time of superconducting nanowire photon counters," Appl. Phys. Lett., 88, 111116, (2006).

23. Kasap, S. O., 2001, Optoelectronics and Photonics: Principles and Practices (Upper Saddle River, NJ: PrenticeHall).

24. Ou, Z. Y. and Mandel, L., Phys. Rev. Lett. 61, 50 (1988). 